\title{
Editorial: Saline Water Evaporation from Porous Media
}

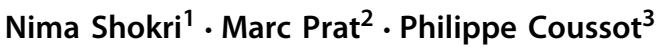 \\ Published online: 16 May 2019 \\ (c) Springer Nature B.V. 2019
}

Saline water evaporation from porous media is a part of our daily experiences, and it is central to many environmental and engineering applications ranging from soil salinization affecting hydrological processes, vegetation, $\mathrm{CO}_{2}$ sequestration to the drying of building materials, pastes and preservation of historical monuments.

Saline water evaporation is influenced by transport properties of porous media, properties of the evaporating solution, external conditions (wind, radiation, ambient temperature and relative humidity) and precipitation of salt crystals. During evaporation, solute is transported, generally via capillary-induced liquid flow, from a wet zone to the evaporation surface resulting in increasing solute concentration close to the vaporization interface. At the same time, diffusion tends to spread the salt homogenously through the entire space. This competition influences the solute distribution during the evaporation process. When salt concentration substantially exceeds the solubility limit, crystals will precipitate by first nucleating and then growing. The structure and patterns of the precipitated salt may evolve as evaporation process proceeds. These dynamic interactions present a challenge to the prediction of saline water evaporation from porous media and interplay among mass and energy exchange, as well as mechanical and other consequences of salt deposition.

This special issue is an outcome of a symposium that was organized in the 9th International Conference of the Interpore society held in Rotterdam, Netherlands, in 2017 that brought together researchers from various disciplines interested in the dynamics of saline water evaporation from porous media. The papers published in this special issue cover a wide range of topics related to saline water evaporation from porous media and the parameters controlling this complex process at different scales by employing a variety of numerical and experimental techniques. It must be noted that although all open questions about the dynamics of saline water evaporation from porous media could not be discussed in this special issue, the papers published here provide the interested readers with a good overview of the physics of this process, the state-of-the-art research on this topic and the tools that are commonly used to investigate saline water evaporation from porous media experimentally and numerically. In the following, we briefly describe the scope of each contribution:

Nima Shokri

nima.shokri@manchester.ac.uk

1 School of Chemical Engineering and Analytical Science, The University of Manchester, Manchester, UK

2 Institut de Mécanique des Fluides de Toulouse, Allée du Professeur Camille Soula, Toulouse, France

3 Laboratoire Navier (ENPC-IFSTTAR-CNRS), Université Paris-Est, 2 Allée Kepler, Champs Sur Marne, France 
Hidri et al. (2018) studied the solute deposition patterns at the surface of porous media during evaporation. Using numerical analysis, they explored the link between the ion concentration spatial variations and porous medium heterogeneities considering strongly anisotropic short-range correlated permeability Gaussian fields. Their numerical analysis illustrated the interaction between the local solute deposition patterns at the surface and transport properties of porous media influencing the dynamics of solute precipitation patterns.

Qazi et al. (2018) investigated the evaporation of $\mathrm{NaCl}$ solutions from porous media in the presence of surfactants. Their analysis illustrated the complex interaction between the presence of surfactant, crystallization process and duration of the evaporation process as well as the evaporative fluxes. They revealed the role of surfactants as a crystallization modifier for the salt during evaporation. Their findings are important for preservation of buildings since surfactants are often used as cleaning agents for porous building materials and the drying kinetics of such materials play an important role on their susceptibility to frost and biological damage.

Derluyn et al. (2018) studied drying dynamics of sodium sulfate and sodium chloride solutions in building materials and quantified the damaging character of anhydrous sodium sulfate and halite precipitation as a result of drying using high-resolution neutron radiography technique. Their analysis provided us with a better physical understanding of the damaging effect of in-pore crystallization of thenardite and halite during drying of porous building materials.

Seck et al. (2018) investigated the impact of the presence of suspended elements such as calcium sulfate ions on the drying characteristics. They used a single capillary for evaporation experiments capable of reproducing some critical aspects of drying of porous media. Their analysis illustrated the strong sensitivity of the drying rate to the composition of the evaporating fluids as well as the wetting characteristics of the porous medium.

Qin et al. (2018) utilized lattice Boltzmann method (LBM) to investigate evaporation of colloidal suspension in two-dimensional (2D) porous media with the associated selfassembled clogging structures. Using LMB, one can deal directly with complex geometry of both the porous medium and the liquid configuration. In this paper, the authors employed an advanced hybrid LBM, combined with an Eulerian approach to account for the colloidal particle transport during evaporation from $2 \mathrm{D}$ porous medium under different scenarios. Their numerical and experimental analysis illustrated the relationship between the properties such as porosity, particle concentration in the evaporating fluid and the evaporation rate during evaporation of colloidal suspension.

Li et al. (2018) performed a series of numerical and experimental analyses to examine the performance of different mathematical formulations used to estimate water evaporation process from bare soils offering a great opportunity to evaluate the formulations, assumptions and boundary conditions used in each model. The model performance was examined using experimental data generated from a soil tank/boundary layer wind tunnel experimental apparatus equipped with a variety of sensors to continuously monitor the parameters required to describe the dynamics of water evaporation from bare soil. Their analysis highlights the need to improve accuracy of the models commonly used to describe the evaporation process.

The last but not the least, we would like to thank the many dedicated reviewers for their professional and constructive comments that improved the quality of the contributions. We are also grateful for professional and timely assistance of the Editorial office of Transport in Porous Media, and we thank the contributing authors to this special issue. 


\section{References}

Derluyn, H., Vontobel, P., Mannes, D., Derome, D., Lehmann, E., Carmeliet, J.: Saline water evaporation and crystallization-induced deformations in building stone: insights from high-resolution neutron radiography. Transp. Porous Media. (2018). https://doi.org/10.1007/s11242-018-1151-X

Hidri, F., Diouf, B., Bouhlila, R., Geoffroy, S., Prat, M.: Stagnation points as loci of solute concentration extrema at the evaporative surface of a random porous medium. Transp. Porous Media. (2018). https:// doi.org/10.1007/s11242-018-1098-y

Li, Z., Vanderborght, J., Smits, K.M.: Evaluation of model concepts to describe water transport in shallow subsurface soil and across the soil-air interface. Transp. Porous Media. (2018). https://doi.org/10.1007/ s11242-018-1144-9

Qazi, M., Bonn, D., Shahidzadeh, N.: Drying of salt solutions from porous media: effect of surfactants. Transp. Porous Media. (2018). https://doi.org/10.1007/s11242-018-1164-5

Qin, F., Mazloomi Moqaddam, A., Kang, Q., Derome, D., Carmeliet, J.: LBM Simulation of self-assembly of clogging structures by evaporation of colloidal suspension in 2D porous media. Transp. Porous Media. (2018). https://doi.org/10.1007/s11242-018-1157-4

Seck, M.D., Keita, E., Coussot, P.: Some observations on the impact of a low-solubility ionic solution on drying characteristics of a model porous medium. Transp. Porous Media. (2018). https://doi.org/10. $1007 / \mathrm{s} 11242-018-1169-0$

Publisher's Note Springer Nature remains neutral with regard to jurisdictional claims in published maps and institutional affiliations. 